\title{
Hiponatremia y lesión pulmonar: ¿una asociación peligrosa en SARS-CoV-2?
}

Hyponatremia and lung injury: a dangerous association in SARS-CoV-2?

Hiponatremia e lesão pulmonar: uma associação perigosa em SARS-CoV-2?

Lyman Ledesma Cervantes, ${ }^{*}$ Jesús Salvador Sánchez Díaz, ${ }^{*}$ Karla Gabriela Peniche Moguel, ${ }^{*}$ Enrique Monares Zepeda, ${ }^{\ddagger}$ Eduardo Alberto González Escudero, ${ }^{*}$ María Verónica Calyeca Sánchez*

\section{RESUMEN}

Introducción: La pandemia por SARS-CoV-2 (Severe Acute Respiratory Syndrome Coronavirus 2) se ha convertido en un hito en la historia moderna de la humanidad, la neumonía grave que ocasiona cursa con niveles elevados de IL-6, la cual estimula el hipotálamo para la secreción de hormona antidiurética y condicionar hiponatremia ( $\mathrm{Na}+$ sérico < $135 \mathrm{mEq} / \mathrm{L}$ ), ésta se presenta en $29 \%$ de los pacientes con diagnóstico de neumonía y se considera un marcador de inflamación, gravedad y pronóstico.

Material y métodos: Estudio de cohorte, ambispectivo, descriptivo, analítico que incluyó pacientes con diagnóstico de neumonía grave por SARS-CoV-2; se clasificaron en dos grupos: grupo 1 (G1) sin hiponatremia y grupo 2 (G2) con hiponatremia, se identificó asociación entre la lesión pulmonar.

Resultados: Se incluyeron 53 pacientes, el G1 incluyó 37 (69.8\%) y el G2 16 $(30.2 \%)$ pacientes, la media de sodio $(\mathrm{Na}+)$ fue $139.86 \pm 3.55$ y $130 \pm 3.29$ para el $\mathrm{G} 1$ y $\mathrm{G} 2$ respectivamente $(\mathrm{p}=0.001)$. De las variables pulmonares la $\mathrm{PaO}_{2}$ $\mathrm{FiO}_{2}$ fue $85.73 \pm 29.93$ y $114 \pm 57.37 \mathrm{mmHg}$, distensibilidad estática de $34.67 \pm$ 8.99 y $31.87 \pm 10.89 \mathrm{cmH}_{2} \mathrm{O}$, para el $\mathrm{G} 1$ y $\mathrm{G} 2$ respectivamente. La mortalidad en el G1 fue $42 \%$ y $75 \%$ en el G2 con OR sodio-mortalidad 3.93 (IC95\% 1.0614.52), $p=0.03$.

Conclusión: Las concentraciones séricas más bajas de $\mathrm{Na}+$ están asociadas a mayor severidad y peores resultados en pacientes con neumonía grave por SARS-CoV-2. Convirtiendo a la medición del sodio sérico en un recurso factible de cuantificación para asociarlo a severidad de lesión pulmonar por SARS-CoV-2 e identificar a los pacientes con mayor riesgo de complicaciones y muerte.

Palabras clave: Neumonía, hiponatremia, SARS-CoV-2, lesión pulmonar.

\section{ABSTRACT}

Introduction: The SARS-CoV-2 (Severe Acute Respiratory Syndrome Coronavirus 2) pandemic has become a milestone in the modern history of mankind, severe pneumonia that causes high levels of IL-6 which stimulates the hypothalamus For antidiuretic hormone secretion and condition hyponatremia (serum $\mathrm{Na}+<135 \mathrm{mEq} / \mathrm{L}$ ), this occurs in 29\% of patients diagnosed with pneumonia and is considered a marker of inflammation, severity and prognosis. Material and methods: Ambispective, descriptive, analytical cohort study that included patients with a diagnosis of severe pneumonia due to SARS-CoV-2; they were classified into two groups: group 1 (G1) without hyponatremia and group 2 (G2) with hyponatremia, an association was identified between lung injury.

Results: 53 patients were included, G1 included 37 (69.8\%) and G2 16 (30.2\%) patients, the mean sodium (Na+) was $139.86 \pm 3.55$ and $130 \pm 3.29$ for $\mathrm{G} 1$ and G2 respectively $(p=0.001)$. Of the pulmonary variables, $\mathrm{PaO}_{2} / \mathrm{FiO}_{2}$ was 85.73 \pm 29.93 and $114 \pm 57.37 \mathrm{mmHg}$, static compliance of $34.67 \pm 8.99$ and $31.87 \pm$ $10.89 \mathrm{cmH}_{2} \mathrm{O}$, for $\mathrm{G} 1$ and $\mathrm{G} 2$ respectively. Mortality in $\mathrm{G} 1$ was $42 \%$ and $75 \%$ in G2 with OR Sodium-Mortality 3.93 (95\% Cl 1.06-14.52), $p=0.03$.

Conclusion: Lower serum $\mathrm{Na}+$ concentrations are associated with greater severity and worse outcomes in patients with severe SARS-CoV-2 pneumonia. Making the measurement of serum sodium a feasible quantification resource to associate it with the severity of lung injury due to SARS-CoV-2 and to identify patients with a higher risk of complications and death.

Keywords: Pneumonia, hyponatremia, SARS-CoV-2, lung injury.

\footnotetext{
* Hospital de Especialidades No. 14, CMN «Adolfo Ruiz Cortines», IMSS, Veracruz, Veracruz. México.

‡ Hospital San Ángel Inn Universidad, Ciudad de México, México.

Recibido: 16/10/2020. Aceptado: 27/10/2020

Citar como: Ledesma CL, Sánchez DJS, Peniche MKG, Monares ZE, González EEA, Calyeca SMV. Hiponatremia y lesión pulmonar: ¿una asociación peligrosa en SARSCoV-2? Med Crit. 2021;35(4):172-175. https://dx.doi.org/10.35366/101154

www.medigraphic.com/medicinacritica
}

\section{RESUMO}

Introdução: A pandemia de SARS-CoV-2 (severe acute respiratory syndrome coronavirus 2) tornou-se um marco na história moderna da humanidade, pneumonia grave que cursa com altos níveis de IL-6 que estimula o hipotálamo para secreção de hormônio antidiurético e condicionar a hiponatremia ( Na+ sérico < $135 \mathrm{mEq} / \mathrm{L}$ ), ocorre em 29\% dos pacientes com diagnóstico de pneumonia e é considerado um marcador de inflamação, gravidade e prognóstico.

Material e métodos: Estudo de coorte ambispectivo, descritivo e analítico que incluiu pacientes com diagnóstico de pneumonia grave por SARS-CoV-2; Foram classificados em dois grupos: grupo 1 (G1) sem hiponatremia e grupo 2 (G2) com hiponatremia, sendo identificada associação entre lesão pulmonar.

Resultados: Foram incluídos 53 pacientes, G1 incluiu 37 (69.8\%) e G2 16 (30.2\%) pacientes, a média de sódio $(\mathrm{Na}+)$ foi de $139.86 \pm 3.55$ e $130 \pm 3.29$ para G1 e G2 respectivamente $(p=0.001)$. Das variáveis pulmonares, $\mathrm{PaO}_{2}$ $\mathrm{FiO}_{2}$ de $85.73 \pm 29.93$ e $114 \pm 57.37 \mathrm{mmHg}$, complacência estática de 34.67 \pm 8.99 e $31.87 \pm 10.89 \mathrm{cmH}_{2} \mathrm{O}$, para $\mathrm{G} 1$ e G2 respectivamente. A mortalidade no G1 foi de 42 e $75 \%$ no G2 com OR sódio-mortalidade 3.93 (IC 95\% 1.0614.52), $p=0.03$.

Conclusão: Baixas concentrações séricas de $\mathrm{Na}$ + estão associadas a maior gravidade e piores resultados em pacientes com pneumonia grave por SARSCoV-2. Tornando a dosagem do sódio sérico um recurso de quantificação viável para associá-la à gravidade da lesão pulmonar por SARS-CoV-2 e identificar pacientes com maior risco de complicações e óbito.

Palavras-chave: Pneumonia, hiponatremia, SARS-CoV-2, lesão pulmonar.

\section{INTRODUCCIÓN}

La pandemia por SARS-CoV-2 se ha convertido en un hito en la historia moderna de la humanidad; la presentación clínica tan variada y su evolución insidiosa hacen esta enfermedad aún más peligrosa y de difícil predicción; $;^{1,2}$ las comorbilidades presentes en el paciente en el momento de la infección se encuentran como factores ya descritos que pueden incidir en la progresión de la enfermedad, sobre todo cuando éstas se encuentran descontroladas o bien sin alcanzar las metas óptimas;; ${ }^{3,4}$ a casi un año de identificada la enfermedad por coronavirus sabemos que la puerta de entrada al organismo es el sistema respiratorio alto, requiriendo del receptor de enzima convertidora de angiotensina 2 para el ingreso a la célula del huésped y así ocasionar daño epitelial y endotelial; también sabemos del protagonismo de la interleucina 6 (IL-6) para ocasionar y perpetuar la injuria tisular de manera sistémica y condicionar a la disfunción orgánica múltiple; ;,6 sin embargo, la presencia de la IL-6 no es exclusiva de esta enfermedad, ya que en modelos humanos de neumonía no viral (comunitaria o asociada a la ventilación mecánica invasiva) se han identificado valores elevados de la concentración de 
la IL-6, por otra parte existe una correlación negativa entre la concentración sérica elevada de la IL-6 y la gravedad de la lesión pulmonar aguda identificada a través de la relación entre la presión arterial de oxígeno y la fracción inspirada de oxígeno $\left(\mathrm{PaO}_{2} / \mathrm{FiO}_{2}\right)$ y la distensibilidad pulmonar, no siendo el caso de las neumonías no virales. ${ }^{7,8}$ La acción de la IL-6 tiene efecto multisistémico, a nivel del sistema nervioso central posee la capacidad de atravesar la barrera hematoencefálica y estimular al hipotálamo para liberar la hormona antidiurética o vasopresina, cuyo resultado de producción alterada será la secreción inapropiada de hormona antidiurética (SIADH) siendo una estimulación no osmótica de la misma y condicionando hiponatremia euvolémica. ${ }^{9}$

La hiponatremia ( $\mathrm{Na}+$ sérico $<135 \mathrm{mEq} / \mathrm{L})$ se presenta en $29 \%$ de los pacientes con diagnóstico de neumonía. De hecho, se considera un marcador de inflamación, gravedad y pronóstico. Los pacientes con neumonía e hiponatremia tienen mayor necesidad de ventilación mecánica invasiva (VMI) y de ingreso a la Unidad de Cuidado Intensivo (UCI). La razón de por qué la neumonía provoca hiponatremia no es del todo clara, pero la secreción inapropiada de hormona antidiurética (SIHAD) es el principal mecanismo involucrado. ${ }^{10}$ Aunque la causa obedece a múltiples factores, existe evidencia de la relación entre la IL-6, inflamación y osmorregulación. La IL-6 se reconoce como el principal mediador inflamatorio de la fase aguda con efectos hematológicos, inmunológicos, endocrinológicos y metabólicos. Los niveles elevados de IL-6 promueven la expresión de proteína $C$ reactiva (PCR) y ferritina, además estimulan la producción de factores protrombóticos como fibrinógeno y dímero D (DD)..$^{11}$ Las infecciones por virus estimulan la síntesis y liberación de IL-6, a decir verdad, está bien documentado que la neumonía grave por SARS-CoV-2 (Severe Acute Respiratory Syndrome Coronavirus 2) cursa con niveles elevados de IL-6, siendo esta un regulador clave de la secreción no osmótica de vasopresina, ${ }^{12}$ lo que podría ocasionar que la enfermedad por coronavirus 2019 (COVID-19) coexista con hiponatremia, la cual podría ser un marcador de gravedad y de mal pronóstico. ${ }^{13}$ Convirtiendo a la medición del sodio sérico en un recurso factible de cuantificación para asociarlo a severidad de lesión pulmonar por SARS-CoV-2 e identificar a los pacientes con mayor riesgo de complicaciones y muerte.

\section{MATERIAL Y MÉTODOS}

Objetivo: Identificar la asociación entre la hiponatremia y la severidad de la lesión pulmonar en la neumonía grave por SARS-CoV-2.

\section{MATERIAL Y MÉTODOS}

Tipo de estudio: estudio de cohorte, ambispectivo, descriptivo, analítico. Criterios de inclusión: pacientes mayores de 18 años con diagnóstico de neumonía grave por SARS-CoV-2, ingresados en la Unidad de Cuidados Intensivos (UCI) del $1^{\circ}$ de mayo al de septiembre de 2020. Criterios de exclusión: pacientes cuyo fallecimiento ocurrió dentro de las primeras ocho horas de ingreso a la UCl. Criterios de eliminación: pacientes que concluyeron su tratamiento médico en otra terapia intensiva. Procedimiento: durante el tiempo comprendido, se revisaron los expedientes médicos de los pacientes que cumplieron con los criterios de inclusión, se recolectaron las variables demográficas, bioquímicas y gasométricas al ingreso de UCl. Se dividió a la población en dos grupos: grupo 1 (G1) con neumonía grave por SARSCoV-2 sin hiponatremia; y grupo 2 (G2) con neumonía grave por SARS-CoV-2 con hiponatremia. Se realizaron pruebas de normalidad, las variables con distribución paramétrica se representaron como media y desviación estándar, mientas que las variables de distribución no paramétrica se representaron como mediana y rangos intercuartilares, las variables cualitativas se representaron como porcentajes. Se realizó prueba T de Student para variables de distribución normal y prueba $U$ de Mann-Whitney para variables de distribución anormal. Para variables cualitativas se utilizó la $\chi^{2}$. Los datos se analizaron con el software SPSS versión 23.

\section{RESULTADOS}

Durante el periodo comprendido se incluyeron 53 pacientes confirmados con SARS-CoV-2, todos con VMI (ventilación mecánica invasiva) desde su ingreso a la $\mathrm{UCl}$, la mortalidad general fue de $53 \%$ (28 pacientes). La tabla 1 muestra las características y variables de ambos grupos. La mortalidad en el G1 fue 42 y $75 \%$ en el G2 con OR sodio-mortalidad 3.93 (IC95\% 1.0614.52), $p=0.03$.

\section{DISCUSIÓN}

No es infrecuente que las patologías pulmonares cursen con hiponatremia secundaria a SIHAD, siendo la neumonía grave por SARS-CoV-2 una de las causas. ${ }^{14}$ La IL-6 incrementa la liberación de hormona antidiurética (HAD) por estimulación directa no osmótica y por daño de la membrana alveolar, resultando en vasoconstricción pulmonar hipóxica. ${ }^{15}$ La propia VMI puede ocasionar hiponatremia secundaria a SIHAD, probablemente por estimulación de los barorreceptores venosos pulmonares en respuesta a la reducción de volumen de sangre efectivo. ${ }^{16} \mathrm{Al}$ respecto, la muestra de $\mathrm{Na}^{+}$sérico de los pacientes en los cuales se documentó hiponatre- 
mia se tomó del catéter venoso central inmediatamente después de la intubación e inicio de la VMI. Berni y colaboradores ${ }^{17}$ documentaron que en pacientes con COVID-19 la IL-6 $>10 \mathrm{pg} / \mathrm{mL}$ se asocia a $\mathrm{Na}^{+}$bajo 128 $\mathrm{mEq} / \mathrm{L}$ (rango $128-145 \mathrm{mEq} / \mathrm{L}$ ) y a menor $\mathrm{PaO}_{2} / \mathrm{FiO}_{2}$ (133.1 \pm 3.5 vs $139.6 \pm 2.4 \mathrm{mmHg}$ ) en comparación con los que tuvieron IL- $6<10 \mathrm{pg} / \mathrm{mL}$. Las correlaciones entre una menor $\mathrm{PaO}_{2} / \mathrm{FiO}_{2}$ y menor $\mathrm{Na}^{+}$fue de 0.6 ( $\mathrm{p}$ $=0.0005)$ y entre menor $\mathrm{Na}^{+}$y una mayor IL-6 fue de $-0.6(p=0006)$. Además, la hiponatremia se asoció con mayor severidad (es decir, ingreso a $\mathrm{UCl}$, ventilación mecánica y muerte) con 53 vs $7 \%, p=0.031$. La regresión logística confirmó OR 14.8 (IC95\% $15 \pm 144.2$, $\mathrm{p}=0.02$ ). Nuestras correlaciones fueron moderadas entre hiponatremia y $\mathrm{PaO}_{2} / \mathrm{FiO}_{2}$ con $0.31(p=0.02)$ y entre hiponatremia y mortalidad con $0.30(p=0.03)$. Los primeros reportes informan que la hiponatremia es la alteración electrolítica más frecuente en los pacientes con COVID-19. Sin embargo, la hiponatremia puede ser ocasionada no solo por SIHAD, sino por ingesta dietética inadecuada o presentación gastrointestinal de la

Tabla 1: Análisis bivariado en pacientes con neumonía grave por SARS-CoV-2 con hiponatremia.

\begin{tabular}{|c|c|c|c|}
\hline Variable & $\begin{array}{c}\text { Sin hiponatremia } \\
n=37 \\
n(\%)\end{array}$ & $\begin{array}{c}\text { Con hiponatremia } \\
n=16 \\
n(\%)\end{array}$ & $\mathrm{p}$ \\
\hline Género & & & 0.57 \\
\hline Hombre & $26(70.3)$ & $10(62.5)$ & \\
\hline Mujer & $11(29.7)$ & $6(37.5)$ & \\
\hline Edad & $63 \pm 13.09$ & $67 \pm 10.65$ & 0.23 \\
\hline Índice de masa corporal & $31.4 \pm 4.97$ & $32.53 \pm 7.53$ & 0.52 \\
\hline \multicolumn{4}{|l|}{ Antecedentes } \\
\hline Tabaquismo & $11(61.1)$ & $7(38.9)$ & 0.320 \\
\hline DM2 & 15 (83.3) & $3(16.7)$ & 0.120 \\
\hline HAS & $21(65.6)$ & $11(34.4)$ & 0.410 \\
\hline Vasopresor & $11(64.7)$ & $6(35.3)$ & 0.570 \\
\hline Troponina I & $0.47 \pm 1.79$ & $0.13 \pm 0.32$ & 0.460 \\
\hline $\mathrm{PaO}^{2} / \mathrm{FiO}^{2}$ & $85.73 \pm 29.93$ & $114 \pm 57.37$ & 0.730 \\
\hline $\mathrm{DP}$ & $15.64 \pm 3.31$ & $15.03 \pm 3.14$ & 0.550 \\
\hline EV & $1.99 \pm 0.47$ & $2.18 \pm 1.00$ & 0.360 \\
\hline PM & $20 \pm 5.70$ & $21.32 \pm 6.10$ & 0.490 \\
\hline Distensibilidad & $34.67 \pm 8.99$ & $31.87 \pm 10.89$ & 0.330 \\
\hline Días de VM & $6.27 \pm 3.90$ & $6.43 \pm 4.38$ & 0.460 \\
\hline Reintubación & $9(24.3)$ & $5(31.25)$ & 0.600 \\
\hline $\mathrm{Cr}$ & $1.43 \pm 3.17$ & $1.29 \pm 0.75$ & 0.860 \\
\hline $\mathrm{Na}^{+}$ & $139.86 \pm 3.35$ & $130 \pm 3.29$ & 0.001 \\
\hline $\mathrm{Cl}^{-}$ & $104 \pm 4.38$ & $95.81 \pm 2.81$ & 0.001 \\
\hline $\mathrm{DD}$ & $3,673 \pm 3,334$ & $2,111 \pm 1,699$ & 0.210 \\
\hline $\mathrm{DHL}$ & $2,259 \pm 1,051$ & $359 \pm 286$ & 0.430 \\
\hline Ferritina & $1,223 \pm 810$ & $1,109 \pm 546$ & 0.550 \\
\hline PCR & $157 \pm 94$ & $183 \pm 109$ & 0.370 \\
\hline SAPS II & $78.61 \pm 8.9$ & $80.43 \pm 8.05$ & 0.380 \\
\hline Días en UCl & $7 \pm 3.64$ & $6.75 \pm 4.09$ & 0.780 \\
\hline Mortalidad en UCI & $16(42.0)$ & $12(75.0)$ & 0.030 \\
\hline
\end{tabular}

$\mathrm{DM} 2$ = diabetes mellitus tipo 2, $\mathrm{HAS}=$ hipertensión arterial sistémica, $\mathrm{PaO}_{2} / \mathrm{FiO}_{2}=$ presión arterial de oxígeno, $\mathrm{DP}=$ driving pressure, $\mathrm{EV}=$ eficiencia ventilatoria, $\mathrm{PM}$ = poder mecánico, $\mathrm{VM}=$ ventilación mecánica, $\mathrm{Cr}=$ creatinina, $\mathrm{Na}^{+}=$sodio, $\mathrm{Cl}=$ cloro, $\mathrm{DD}=$ dímero $\mathrm{D}, \mathrm{DHL}=$ deshidrogenasa láctica, $\mathrm{PCR}=$ proteína $\mathrm{C}$ reactiva, SAPS II = simplified acute physiology score $I I, \mathrm{UCl}=$ Unidad de Cuidado Intensivo. enfermedad. ${ }^{18}$ Otras alteraciones electrolíticas frecuentes son hipopotasemia e hipocalcemia con implicaciones clínicas significativas. ${ }^{19}$

\section{CONCLUSIÓN}

Consideramos que las concentraciones séricas más bajas de $\mathrm{Na}+$ están asociadas a mayor severidad y peores resultados en pacientes con neumonía grave por SARS-CoV-2. La hiponatremia podría ser un subrogado de la IL-6, por lo tanto, reflejar mayor gravedad y ser considerado como un factor independiente de mortalidad en esta población de pacientes; sin embargo, se necesitan más estudios para afirmar esta conclusión.

\section{REFERENCIAS}

1. Letko M, Munster V. Functional assessment of cell entry and receptor usage for lineage $B \beta$-coronaviruses, including 2019nCoV. Nature Microbiology. doi: 10.1101/2020.01.22.915660.

2. Hu B, Guo H, Zhou P, et al. Characteristics of SARS-CoV-2 and COVID-19. Nat Rev Microbiol. 2020;1-14. doi: 10.1038/s41579020-00459-7.

3. Cui J, Li F, Shi ZL. Origin and evolution of pathogenic coronaviruses. Nat Rev Microbiol. 2019;17:181-192. doi: 10.1038/s41579-018-0118-9.

4. Han Q, Lin Q, Jin S, You L. Coronavirus 2019-nCoV: a brief perspective from the front line. J Infect. 2020;80(4):373-377. doi: 10.1016/j.jinf.2020.02.010.

5. Mehta P, McAuley D, Brown M, et al. COVID-19: consider cytokine storm syndromes and immunosuppression. Lancet. 2020;395(10229):1033-1034. doi: 10.1016/S01406736(20)30628-0.

6. Yang L, Liu S, Liu J, et al. COVID-19: immunopathogenesis and immunotherapeutics. Signal Transduction and Targeted Therapy. 2020:128. doi: org/10.1038/s41392-020-00243-2.

7. Stukas S, Hoiland R, Cooper J, et al. The association of inflammatory cytokines in the pulmonary pathophysiology of respiratory failure in critically ill patients with coronavirus disease 2019. Crit Care Expl. 2020;2:e0203. doi: 10.1097/ CCE.0000000000000203.

8. Aziz M, Aziz M, Fatima R, et al. Elevated interleukin-6 and severe COVID-19: a meta-analysis. J Med Virol. 2020;92:2283-2285.

9. Habib MB, Sardar S, Sajid J. Acute symptomatic hyponatremia in setting of SIADH as an isolated presentation of COVID-19. IDCases. 2020;21:e00859. doi: 10.1016/j.idcr.2020.e00859.

10. Edmonds ZV. Hyponatremia in pneumonia. J Hosp Med. 2012;7 Suppl 4:S11-S13. doi: 10.1002/jhm.1933.

11. Swart RM, Hoorn EJ, Betjes MG, Zietse R. Hyponatremia and inflammation: the emerging role of interleukin-6 in osmoregulation. Nephron Physiol. 2011;118(2):45-51. doi: $10.1159 / 000322238$.

12. Kox M, Waalders NJB, Kooistra EJ, Gerretsen J, Pickkers P. Cytokine levels in critically ill patients with COVID-19 and other conditions. JAMA. 2020;324(15):1565-1567. doi: 10.1001/ jama.2020.17052.

13. Aggarwal S, Garcia-Telles N, Aggarwal G, Lavie C, Lippi G, Henry BM. Clinical features, laboratory characteristics, and outcomes of patients hospitalized with coronavirus disease 2019 (COVID-19): early report from the United States. Diagnosis. 2020;7:91-96. doi: 10.1515/dx-2020-0046.

14. Yousaf Z, Al-Shokri SD, Al-Soub H, Mohamed MFH. COVID-19associated SIADH: a clue in the times of pandemic! Am J Physiol Endocrinol Metab. 2020;318(6):E882-E885. doi: 10.1152/ ajpendo.00178.2020.

15. Sheikh M, Ahmad E, Jeelani H, et al. COVID-19 pneumonia: an emerging cause of syndrome of inappropriate antidiuretic hormone. Cureus. 2020;12(6):e8841. doi: 10.7759/cureus.8841. 
16. Khalangot M. COVID-19 and SIADH relations: impact of the positive pressure ventilation. Am J Physiol Endocrinol Metab. 2020;319(1):E196. doi: 10.1152/ajpendo.00288.2020.

17. Berni A., Malandrino D., Parenti, G, et al. Hyponatremia, IL-6, and SARS-CoV-2 (COVID-19) infection: may all fit together? J Endocrinol Invest. 2020:1137-1139. doi: org/10.1007/s40618-020-01301-w.

18. Królicka AL, Kruczkowska A, Krajewska M, Kusztal MA. Hyponatremia in infectious diseases-a literature review. Int $J$ Environ Res Public Health. 2020;17(15):5320. doi: 10.3390/ijerph17155320.

19. Lippi G, South AM, Henry BM. Electrolyte imbalances in patients with severe coronavirus disease 2019 (COVID-19). Ann Clin Biochem. 2020;57(3):262-265. doi: 10.1177/0004563220922255.
Patrocinios: El presente trabajo no recibió patrocinios, sólo se utilizaron recursos propios de nuestro hospital.

Conflicto de intereses: Los autores declaramos no tener conflicto de intereses.

Correspondencia:

Dr. Jesús Salvador Sánchez Díaz

E-mail: drsalvadorsanchezdiaz@gmail.com 